\title{
Lesson of the month 1: Acute flecainide overdose and the potential utility of lipid emulsion therapy
}

\author{
Authors: Omar Mukhtar, ${ }^{\mathrm{A}}$ John RH Archer, ${ }^{\mathrm{B}}$ Paul I Dargan ${ }^{\mathrm{C}}$ and David M Wood ${ }^{\mathrm{D}}$
}

\begin{abstract}
Lipid-emulsion therapy (Intralipid ${ }^{\circledR}$ ) has been advocated as a potential treatment for the management of cardio-toxicity arising from lipid-soluble drugs, particularly those acting upon sodium channels. This, on the basis of a number of ex vivo studies and animal models, suggests that partitioning a drug into lipid could alter its pharmacokinetics and result in significant clinical improvements. Its subsequent use in clinical case series has been seen as confirmation of this mechanism of action. While there are undoubtedly instances where lipid emulsion therapy has been associated with a desirable outcome in humans, as described in this case report, clinicians are reminded that they should not attribute causality, on this basis alone.
\end{abstract}

KEYWORDS: Flecainide, toxicity, overdose, lipid emulsion therapy, Intralipid

\section{Background}

Lipid emulsion therapy (Intralipid ${ }^{\circledR}$ ) is an established treatment for the management of acute toxicity resulting from local anaesthetics. ${ }^{1}$ In recent years, it has increasingly been advocated for the management of severe cardiovascular toxicity arising from a variety of other lipid-soluble drugs eg flecainide. ${ }^{2,3}$

We report upon an instance of acute flecainide toxicity in a child where intravenous lipid emulsion therapy (ILE) was associated with a favourable outcome, with analytical confirmation of flecainide ingestion and the impact of this treatment on flecainide concentrations. We also discuss two previous instances where ILE has been associated with a desirable outcome in humans, when exposed to supratherapeutic

Authors: A SpR in clinical pharmacology and therapeutics, King's Health Partner, King's College Hospital, London, UK; ${ }^{B}$ consultant physician and clinical toxicologist, Clinical Toxicology, Guy's and St Thomas' NHS Foundation Trust and King's Health Partners, London, UK; ' Consultant physician and clinical toxicologist, Clinical Toxicology, Guy's and St Thomas' NHS Foundation Trust and King's Health Partners, London, UK, and King's College London, London, UK; ' Consultant physician and clinical toxicologist, Clinical Toxicology, Guy's and St Thomas' NHS Foundation Trust and King's Health Partners, London, UK, and King's College London, London, UK concentrations of flecainide. By critically evaluating our experience along with these previous case reports, we remind clinicians that a causal benefit should not be presumed.

\section{Case report}

A 13-year-old girl attended her local emergency department (ED) 90 minutes after consuming $25 \mathrm{mg}$ bisoprolol, $900 \mathrm{mg}$ flecainide and $225 \mathrm{mg}$ aspirin. Her weight was $45 \mathrm{~kg}$ and she had no significant medical history. On arrival, she was alert (GCS 15/15) with a normal respiratory rate (12 breaths per minute) and oxygen saturations of $100 \%$ on room air; although her heart rate was $65 \mathrm{bpm}$, she was found to be hypotensive (70/39 mmHg). The initial 12-lead ECG demonstrated a sinus rhythm (ventricular rate $70 \mathrm{bpm}$ ), first-degree AV nodal blockade (PR interval $215 \mathrm{~ms}$ ), right bundle branch block (QRS duration $164 \mathrm{~ms}$ ) and a $\mathrm{QT}_{\mathrm{c}}$ of $452 \mathrm{~ms}$. Her serum sodium was $137 \mathrm{mmol} / \mathrm{l}$, potassium $3.7 \mathrm{mmol} / \mathrm{l}$ and magnesium $0.83 \mathrm{mmol} / \mathrm{l}$; a four-hour salicylate level was $<50 \mathrm{mg} / \mathrm{l}$, while her four-hour paracetamol level was $<10 \mathrm{mg} / \mathrm{l}$. The venous $\mathrm{pH}$ was 7.55 , bicarbonate $24.1 \mathrm{mmol} / \mathrm{l}$ and $\mathrm{BE}+3.0$. Two $900 \mathrm{ml}$ boluses $(20 \mathrm{ml} / \mathrm{kg})$ of $0.9 \%$ sodium chloride solution were given and advice sought from the medical toxicology service.

The clinical toxicologist on call recommended intravenous glucagon therapy in view of her bisoprolol ingestion; however $4 \mathrm{mg}$ was given intramuscularly. $8.4 \%$ intravenous sodium bicarbonate was also advised in an effort to maintain a $\mathrm{pH}$ of 7.45-7.50, along with an infusion of magnesium sulphate $(20 \mathrm{mmol})$. Despite these interventions, the patient had a VF arrest one hour after presenting to the ED. Following successful cardiopulmonary resuscitation, she was transferred to a tertiary care centre, intubated, ventilated and with ionotropic support in situ. Prior to transfer and immediately after the return of cardiac output, a $70 \mathrm{ml}$ bolus of $20 \%$ ILE $(1.5 \mathrm{ml} / \mathrm{kg})$ was administered, followed by a further $225 \mathrm{ml}(0.25 \mathrm{ml} / \mathrm{kg} / \mathrm{min})$ over the next 20 minutes.

For the next 8 hours, severe cardiovascular instability was observed with the cardiac rhythm frequently alternating between a sinus tachycardia with right bundle branch block, Torsades de Pointes, a Brugada-like syndrome, coarse VT and ventricular standstill. Intravenous bicarbonate and ionotropes were continued throughout this period; although a temporary transvenous pacing wire was inserted, this was never utilised. Twelve hours after the initial presentation, cardiac stability returned; serial 12-lead ECGs demonstrated sinus rhythm, a 
Fig 1. Serum flecainide concentrations and ECG features post overdose. $A=$ presenta tion to ED; PR interval 215 msec; QRS duration 164 msec; QTC duration $452 \mathrm{msec}$. $B=V F$ arrest $20 \%$ Intralipid $1.5 \mathrm{ml} / \mathrm{kg}$ bolus followed by $0.25 \mathrm{ml} / \mathrm{kg} / \mathrm{min}$ (over 20 mins). $C=$ PR interval 185 msec; QRS duration 124 msec; QTc duration 601 msec. $\mathrm{D}=\mathrm{PR}$ interval 185 msec; QRS duration 124 msec; QTc duration $601 \mathrm{msec}$.

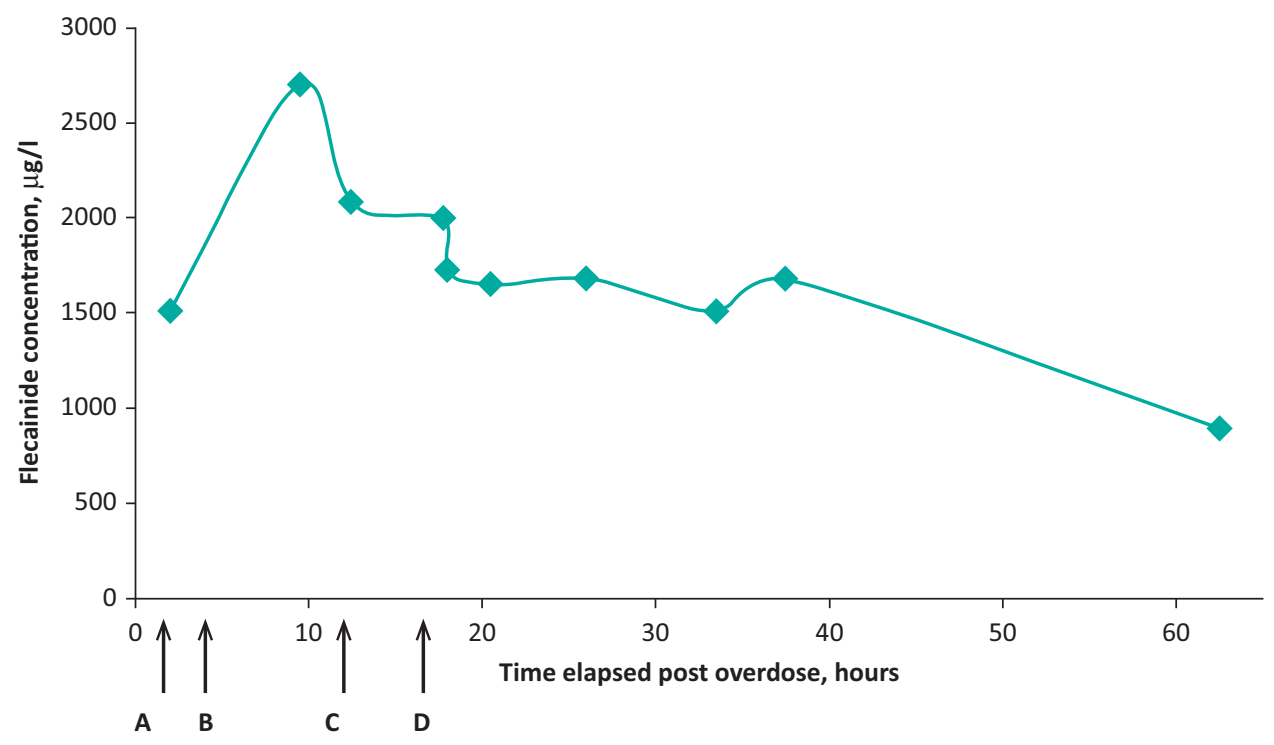

QRS duration of $<120 \mathrm{~ms}$ and $\mathrm{a} \mathrm{QT}_{\mathrm{c}}$ of $<450 \mathrm{~ms}$. The pacing wire was withdrawn, the inotropes weaned and the patient successfully extubated. A degree of ischaemic liver injury was noted, with the serum aspartate transaminase (AST) and alanine transaminase (ALT) peaking at 3,781 and 3,110 IU/l respectively, 60 hours post overdose. With progressive improvement in these indices and after a period of psychiatric assessment, the patient was discharged home with no obvious sequelae.

\section{Flecainide toxicokinetics}

The total flecainide concentration 90 minutes post overdose was $1,512 \mu \mathrm{g} / \mathrm{l}$, increasing to $2,699 \mu \mathrm{g} / \mathrm{l} 9$ hours post ingestion. At $12,24,60$ and $140 \mathrm{~h}$ this had fallen to 2,075, 1,680, 895 and $<100 \mu \mathrm{g} / \mathrm{l}$ respectively (Fig 1 ). With an elimination halflife of 32 hours, the use of Intralipid did not appear to have significantly altered the toxicokinetic profile of flecainide; previous toxicokinetic reports have established the elimination half-life of flecainide to be $28-30$ h. ${ }^{3,4}$ However, such toxicokinetic data should be interpreted with caution, as total, not free flecainide concentrations were measured, and ILE was administered during the likely absorption of flecainide.

\section{Discussion}

The reported mortality rate from overdoses of class Ic Vaughan Williams anti-arrhythmics is $22.5 \% .^{5}$ Accepted treatments include aggressive fluid resuscitation, the administration of hypertonic sodium bicarbonate and intravenous magnesium. ${ }^{6,7}$

Equally, ILE has been heralded a lifesaving treatment in the context of flecainide toxicity. However, the evidence for this assertion is poor; animal data from Cave et al, failed to demonstrate the efficacy of ILE in this setting, when compared with hypertonic sodium bicarbonate. ${ }^{8}$ Moreover, while ILE may act as a 'lipid sink', removing lipid-soluble drugs from the serum and thus limiting any clinical sequelae, this mechanism of action is, at best, putative. ${ }^{3,9}$
In that vein, a number of human case reports have also been cited as evidence for the efficacy of ILE in life-threatening flecainide toxicity. ${ }^{10,11}$ In the first instance, a 72-year-old female developed haemodynamic compromise after ingesting 1,500 mg flecainide, $150 \mathrm{mg}$ oxazepam and $1.5 \mathrm{mg}$ thyroxine. ${ }^{10} \mathrm{On}$ arrival in the $\mathrm{ED}$, her blood pressure was $70 / 50 \mathrm{mmHg}$, heart rate 55 bpm, QRS >200 ms and QT interval 'prolonged'. Following initial resuscitation with intravenous fluids, sodium bicarbonate and adrenaline, lipid emulsion therapy was administered. Thirty minutes later, the authors reported that 'haemodynamic stabilisation allowed a dramatic decrease in the dose of epinephrine (adrenaline)'. However, the exact haemodynamic parameters pre- and post-lipid emulsion therapy were not reported nor were details of flecainide concentrations. The second case report was of a 51-year-old male who ingested 2,500mg of flecainide 90 minutes prior to his arrival in an ED; at presentation his systolic blood pressure was $140 \mathrm{mmHg}$, heart rate $44 \mathrm{bpm}$, QRS $162 \mathrm{~ms}$ and $\mathrm{QT}_{\mathrm{c}} 427 \mathrm{~ms} .{ }^{11} \mathrm{He}$ was then treated with intravenous fluids, sodium bicarbonate, atropine, magnesium and lipid emulsion therapy. While the authors attribute the successful outcome to the use of ILE, the associated graphic suggests that the QRS duration was beginning to 'normalise' prior to the administration of ILE, casting doubt over the impact of ILE in this instance too.

Although we describe a third positive outcome following the use of ILE in the context of a life-threatening flecainide overdose, we remain cautious when attributing therapeutic efficacy. ILE was not administered in isolation of other antidotes/treatments, cardiovascular instability continued to be observed for several hours after its administration and the elimination half-life of flecainide appeared unchanged. Although ILE may have removed flecainide from the circulation into a 'lipid sink', thereby preventing it from causing further cardiovascular toxicity, such an analysis is speculative as we, along with others, are unable to measure free flecainide concentrations. ${ }^{10,11}$ Thus, while ILE may have a role as a 'rescue' therapy in this setting, we would counsel clinicians against the presumption of causality. 


\section{References}

1 Cave G, Harrop-Griffiths W, Harvey M et al. AAGBI safety guideline: management of severe local anaesthetic toxicity. Available online at www.aagbi.org/sites/default/files/la_toxicity_2010_0.pdf [Accessed 19 March 2015].

2 American College of Medical Toxicology. ACMT position statement: interim guidance for the use of lipid resuscitation therapy. J Med Toxicol 2011;7:81-2.

3 Jamaty C, Bailey B, Laroque A et al. Lipid emulsions in the treatment of acute poisonings: a systematic review of human and animal studies. Clin Toxicol 2010;48:1-27.

4 Wood DM, Angel T, Dargan PI, Jones AL. Flecanide toxicity: a case report with toxicokinetic data. Br J Clin Pharmacol 2003;55:431-2.

5 Koppel C, Oberdisse U, Heinemeyer G. Clinical course and outcome in class Ic antiarrhythmic overdose. J Toxicol Clin Toxicol 1990;28:433-44.

6 Roden DM, Woosley RL. Drug therapy. Flecainide. N Engl J Med 1986;315:36-41.
7 Cheung ITF, Man CY. Review on flecainide poisoning. Hong Kong J Emerg Med 2002;9:150-3.

8 Cave G, Harvey M, Quinn P, Heys D. Hypertonic sodium bicarbonate versus intravenous lipid emulsion in a rabbit model of intravenous flecainide toxicity: no difference, no sink. Clin Toxicol 2013;51:394-7.

9 Rothschild L, Bern S, Oswald S, Weinberg G. Intravenous lipid emulsion in clinical toxicology. Scand J Trauma Resusc Emerg Med 2002;18:51.

10 Ellsworth S, Stellpflug SJ, Cole JB et al. A life threatening flecainide overdose successfully treated with intravenous lipid emulsion. Pacing Clin Electrophysiol 2013;36:e87-9.

11 Moussot PE, Marhar F, Minville V et al. Use of 20\% lipid emulsion for treatment of a voluntary intoxication with flecainide with refractory shock. Clin Toxicol 2011;49:514.

Address for corresponding author: Dr O Mukhtar, King's College Hospital, Denmark Hill, London SE5 9RS, UK. Email: o.mukhtar@nhs.net

\section{Applications now open MSc in Medical Leadership 2015/16}

Designed to equip doctors with the practical skills and expertise required to lead clinical and multi-professional teams at all levels, this part-time modular programme incorporates a series of case studies and exclusive lunchtime seminars delivered by high-profile medical leaders. Applications close 1 July 2015

Visit our website to hear more feedback from our students: www.rcplondon.ac.uk/medicalleadership 